\title{
ON A CHARACTERISTIC PROPERTY OF FINITE-DIMENSIONAL BANACH SPACES*
}

\author{
ANTONÍN SLAVÍK \\ Faculty of Mathematics and Physics, Charles University, Sokolovská 83, \\ 18675 Praha 8, Czech Republic \\ e-mail:slavik@karlin.mff.cuni.cz
}

(Received 26 January 2010; revised 11 August 2010; accepted 21 September 2010; first published online 10 March 2011)

\begin{abstract}
This paper is inspired by a counter example of J. Kurzweil published in [5], whose intention was to demonstrate that a certain property of linear operators on finite-dimensional spaces need not be preserved in infinite dimension. We obtain a stronger result, which says that no infinite-dimensional Banach space can have the given property. Along the way, we will also derive an interesting proposition related to Dvoretzky's theorem.
\end{abstract}

2010 Mathematics Subject Classification. 47A30, 47A63, 46B07, 15A45.

1. Introduction. Let $X$ be a real Banach space and $\mathcal{L}(X)$ the space of all bounded linear operators on $X$. Let $I$ denote the identity operator. We say that $X$ has the property $(\mathrm{JK})$, if the following statement is true:

For every $\varepsilon>0$, there exists $\delta>0$ such that if $n \in \mathbb{N}$ and $Z_{1}, \ldots, Z_{n} \in \mathcal{L}(X)$ are operators satisfying

$$
\left\|\left(I+Z_{j_{p}}\right)\left(I+Z_{j_{p-1}}\right) \cdots\left(I+Z_{j_{1}}\right)-I\right\| \leq \delta
$$

for every $p \in\{1, \ldots, n\}$ and every $p$-tuple $1 \leq j_{1}<j_{2}<\cdots<j_{p} \leq n$, then

$$
\sum_{j=1}^{n}\left\|Z_{j}\right\| \leq \varepsilon
$$

In short, the property $(\mathrm{JK})$ guarantees that the sum $\sum_{j=1}^{n}\left\|Z_{j}\right\|$ is small whenever all the 'products' $\left(I+Z_{j_{p}}\right)\left(I+Z_{j_{p-1}}\right) \cdots\left(I+Z_{j_{1}}\right)$ are close to the identity operator.

The property (JK) plays an important role in product-integration theory (see [3,

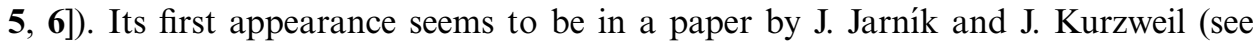
[3]), who have investigated the case $X=\mathbb{R}^{n}$ and $\mathcal{L}(X)=\mathbb{R}^{n \times n}$. They showed that this space possesses the property $(\mathrm{JK})$; since all norms on a finite-dimensional space are equivalent, their result implies that every finite-dimensional space has the property (JK).

On the other hand, the paper of Š. Schwabik (see [5]) contains an example of J. Kurzweil, which shows that the space $c_{0}$ does not have the property (JK). Our main

\footnotetext{
* Supported by grant KJB101120802 of the Grant Agency of the Academy of Sciences of the Czech Republic, and by grant MSM 0021620839 of the Czech Ministry of Education.
} 
goal is to investigate other infinite-dimensional Banach spaces and see whether they have the property $(\mathrm{JK})$.

2. Main results. The argument that lies at the core of J. Kurzweil's example can be stated as follows:

LEMMA 1. Let $X$ be a Banach space and $\left\{c_{n}\right\}_{n=1}^{\infty}$ a sequence of positive numbers such that $\lim _{n \rightarrow \infty}\left(c_{n} / n\right)=0$. Assume that for every $n \in \mathbb{N}$, there exists operators $E_{1}, \ldots, E_{n} \in$ $\mathcal{L}(X)$ satisfying the following conditions:

(i) $\left\|E_{i}\right\| \geq 1$ for every $i \in\{1, \ldots, n\}$,

(ii) $\left\|\sum_{k=1}^{p} E_{j_{k}}\right\| \leq c_{n}$ for every $p \in\{1, \ldots, n\}$ and every $p$-tuple $1 \leq j_{1}<j_{2}<\cdots<$ $j_{p} \leq n$,

(iii) $E_{i} E_{j}=0$ whenever $i>j$.

Then, the space $X$ does not have the property (JK).

Proof. Assume for contradiction that $X$ has the property (JK). Choose an arbitrary $\varepsilon>0$ and let $\delta>0$ be the corresponding constant from the definition of the property (JK). Put $Z_{i}=\delta / c_{n} \cdot E_{i}$ for $i \in\{1, \ldots, n\}$. It follows from the assumptions that for every $p \in\{1, \ldots, n\}$ and every $p$-tuple $1 \leq j_{1}<j_{2}<\cdots<j_{p} \leq n$, we have

$$
\left\|\left(I+Z_{j_{p}}\right)\left(I+Z_{j_{p-1}}\right) \cdots\left(I+Z_{j_{1}}\right)-I\right\|=\left\|\sum_{k=1}^{p} Z_{j_{k}}\right\|=\delta / c_{n} \cdot\left\|\sum_{k=1}^{p} E_{j_{k}}\right\| \leq \delta .
$$

Thus, by taking $n$ such that $c_{n} / n<\delta / \varepsilon$ (remember that $\lim _{n \rightarrow \infty}\left(c_{n} / n\right)=0$ ), we have found $n$ operators $Z_{1}, \ldots, Z_{n}$ such that

$$
\left\|\left(I+Z_{j_{p}}\right)\left(I+Z_{j_{p-1}}\right) \cdots\left(I+Z_{j_{1}}\right)-I\right\| \leq \delta
$$

for every $p$-tuple $1 \leq j_{1}<j_{2}<\cdots<j_{p} \leq n$, but

$$
\sum_{k=1}^{n}\left\|Z_{j}\right\| \geq n \delta / c_{n}>\varepsilon
$$

a contradiction. Therefore, $X$ does not have the property (JK).

In the following example, we use the previous Lemma to prove that the space $c_{0}$ does not have the property (JK); this is the example of J. Kurzweil (see [5]).

EXAMPLE 2. Let $X=c_{0}$, i.e. the space of all real sequences $\left\{a_{n}\right\}_{n=1}^{\infty}$ such that $\lim _{n \rightarrow \infty} a_{n}=0$. The space is equipped with the norm

$$
\left\|\left\{a_{i}\right\}_{i=1}^{\infty}\right\|=\sup _{i \in \mathbb{N}}\left|a_{i}\right| .
$$

Given $n \in \mathbb{N}$, we define operators $E_{1}, \ldots, E_{n} \in \mathcal{L}(X)$ in the following way:

$$
E_{k}\left(\left\{a_{i}\right\}_{i=1}^{\infty}\right)=\left\{b_{i}\right\}_{i=1}^{\infty},
$$

where $b_{i}=0$ for $i \neq 2 k-1$ and $b_{2 k-1}=a_{2 k}$, i.e. the operator $E_{k}$ sets all components of the given sequence except the $2 k$-th one to zero, and then shifts the result to the 
left. It is easy to see that $E_{i} E_{j}=0$ when $i \neq j,\left\|E_{i}\right\|=1$ for every $i \in\{1, \ldots, n\}$, and $\left\|\sum_{k=1}^{p} E_{j_{k}}\right\|=1$ for every $p \in\{1, \ldots, n\}$ and every $p$-tuple $1 \leq j_{1}<j_{2}<\cdots<j_{p} \leq n$. Thus, by Lemma 1 , the space $c_{0}$ does not have the property (JK).

A close inspection of the previous example reveals that a similar argument works in a more general setting. As a prerequisite, we need the following projection theorem of Kadets and Snobar. Recall that a projection of a space $X$ onto a subspace $V$ is a linear mapping $P: X \rightarrow V$ such that $P^{2}=P$ and the range of $P$ is $V$.

THEOREM 3 (Kadets-Snobar theorem). Let $X$ be a Banach space and $V$ a finitedimensional subspace of $X$. Then, there exists a projection $P$ of $X$ onto $V$ such that $\|P\| \leq \sqrt{\operatorname{dim} V}$.

Proof. See the original paper [4] or the monograph [1].

Note the following obvious fact: Since the range of $P$ is $V$, every $v \in V$ can be written as $v=P(w)$ for some $w \in X$. It follows that $P(v)=P^{2}(w)=P(w)=v$, i.e. the restriction of $P$ to $V$ is the identity operator.

LemMA 4. Let $X$ be a Banach space and $c>0, d>0$ two constants such that for every $m \in \mathbb{N}$, there exist vectors $x_{1}, \ldots, x_{m} \in X$ such that

(i) $\left\{x_{1}, \ldots, x_{m}\right\}$ is a linearly independent set,

(ii) $\left\|x_{i}\right\|=1$ for every $i \in\{1, \ldots, m\}$,

(iii) $\left\|\sum_{i \in I} \alpha_{i} x_{i}\right\| \leq c\left\|\sum_{i=1}^{m} \alpha_{i} x_{i}\right\|$ for every $I \subset\{1, \ldots, m\}$ and $\alpha_{1}, \ldots, \alpha_{m} \in \mathbb{R}$,

(iv) $\left\|\sum_{i=1}^{m-1} \alpha_{i+1} x_{i}\right\| \leq d\left\|\sum_{i=1}^{m} \alpha_{i} x_{i}\right\|$ for every $m$-tuple $\alpha_{1}, \ldots, \alpha_{m} \in \mathbb{R}$.

Then, the space $X$ does not have the property $(\mathrm{JK})$.

Proof. Let $n \in \mathbb{N}$ be a given number. In order to prove the statement, we are going to construct operators $E_{1}, \ldots, E_{n}$ satisfying the assumptions of Lemma 1.

Taking $m=2 n$, let $x_{1}, \ldots, x_{2 n} \in X$ be some vectors having the properties $(i)-(i v)$. Let $V$ be the $2 n$-dimensional subspace of $X$ spanned by $x_{1}, \ldots, x_{2 n}$. For $k \in\{1, \ldots, n\}$, we define the operator $E_{k}^{\prime}: V \rightarrow V$ by

$$
E_{k}^{\prime}\left(\sum_{i=1}^{2 n} \alpha_{i} x_{i}\right)=\alpha_{2 k} x_{2 k-1}
$$

It is clear that $\left\|E_{k}^{\prime}\right\| \geq\left\|E_{k}^{\prime}\left(x_{2 k}\right)\right\|=\left\|x_{2 k-1}\right\|=1$. On the other hand, the assumption (iii) implies

$$
\left\|\alpha_{2 k} x_{2 k-1}\right\|=\left|\alpha_{2 k}\right|=\left\|\alpha_{2 k} x_{2 k}\right\| \leq c\left\|\sum_{i=1}^{2 n} \alpha_{i} x_{i}\right\|,
$$

i.e. $\left\|E_{k}^{\prime}\right\| \leq c$ for every $k \in\{1, \ldots, n\}$. Now, consider a $p \in\{1, \ldots, n\}$ and a $p$-tuple $1 \leq j_{1}<\overline{j_{2}}<\cdots<j_{p} \leq n$. Take an arbitrary $x \in V$ with $\|x\|=1$, and write it as $x=$ $\sum_{i=1}^{2 n} \alpha_{i} x_{i}$. Then,

$$
\left\|\left(\sum_{k=1}^{p} E_{j_{k}}^{\prime}\right)\left(\sum_{i=1}^{2 n} \alpha_{i} x_{i}\right)\right\|=\left\|\sum_{k=1}^{p} \alpha_{2 j_{k}} x_{2 j_{k}-1}\right\| \leq c d .
$$


(We have used assumptions (iii) and (iv).) Therefore,

$$
\left\|\sum_{k=1}^{p} E_{j_{k}}^{\prime}\right\| \leq c d
$$

Finally, it is clear that $E_{i}^{\prime} E_{j}^{\prime}=0$, whenever $i \neq j$.

Now, let $P$ be a projection of $X$ onto $V$ such that $\|P\| \leq \sqrt{2 n}$. We define operators $E_{1}, \ldots, E_{n}: X \rightarrow X$ by

$$
E_{k}(x)=E_{k}^{\prime}(P(x)), \quad x \in X, \quad k \in\{1, \ldots, n\} .
$$

These operators are linear and bounded, because

$$
\left\|E_{k}\right\| \leq\left\|E_{k}^{\prime}\right\| \cdot\|P\| \leq c \sqrt{2 n}, \quad k \in\{1, \ldots, n\} .
$$

Since $E_{k}(x)=E_{k}^{\prime}(x)$ for $x \in V$, we have a lower bound

$$
\left\|E_{k}\right\| \geq 1, \quad k \in\{1, \ldots, n\} .
$$

For $i \neq j$ and $x \in X$, we have

$$
E_{i} E_{j}(x)=E_{i}^{\prime}\left(P\left(E_{j}^{\prime}(P(x))\right)=E_{i}^{\prime}\left(E_{j}^{\prime}(P(x))\right)=0 .\right.
$$

Finally, if $x \in X$ and $\|x\|=1$, then $\|P(x)\| \leq \sqrt{2 n}$, and thus,

$$
\left\|\sum_{k=1}^{p} E_{j_{k}}(x)\right\|=\left\|\left(\sum_{k=1}^{p} E_{j_{k}}^{\prime}\right)(P(x))\right\| \leq \sqrt{2 n} \cdot\left\|\sum_{k=1}^{p} E_{j_{k}}^{\prime}\right\| \leq c d \sqrt{2 n}
$$

for every $p \in\{1, \ldots, n\}$ and every $p$-tuple $1 \leq j_{1}<j_{2}<\cdots<j_{p} \leq n$, which means that

$$
\left\|\sum_{k=1}^{p} E_{j_{k}}\right\| \leq c d \sqrt{2 n}
$$

The following examples show that certain familiar infinite-dimensional Banach spaces do not have the property (JK). In each case, we suggest a choice of vectors $x_{1}, \ldots, x_{m}$ (where $m \in \mathbb{N}$ is arbitrary) and leave it up to the reader to check that these vectors satisfy the assumptions of Lemma 4.

EXAMPLE 5. For $X=\ell^{p}, p \in[1, \infty)$, there is a natural choice: Let

$$
x_{k}=\left\{\delta_{k n}\right\}_{n=1}^{\infty}, \quad k \in\{1, \ldots, m\},
$$

where $\delta_{k n}$ denotes the Kronecker symbol. This choice also works when $X=\ell^{\infty}, X=c$ or $X=c_{0}$.

EXAmple 6. Let $X=\mathcal{L}^{p}([a, b])$, where $p \in[1, \infty)$. Then, we can choose

$$
x_{k}=\frac{m}{b-a} \cdot f_{k}, \quad k \in\{1, \ldots, m\}
$$


where $f_{k}:[a, b] \rightarrow \mathbb{R}$ is the characteristic function of interval $(a+(k-1)(b-$ a) $/ m, a+k(b-a) / m)$.

EXAMPLE 7. When $X=\mathcal{C}([a, b])$, we can take

$$
x_{k}=f_{k}, \quad k \in\{1, \ldots, m\},
$$

where $f_{k}:[a, b] \rightarrow \mathbb{R}$ is a function, which is zero outside $I=(a+(k-1)(b-a) / m, a+$ $k(b-a) / m)$, it equals 1 at the midpoint of $I$ and is linear on both halves of $I$. This choice also works when $X=\mathcal{L}^{\infty}([a, b])$.

It should be clear that whenever an infinite-dimensional Banach space $X$ contains an isometric copy of one of the spaces mentioned in the previous examples, then $X$ does not have the propery (JK). Unfortunately, not every Banach space contains an isometric copy of $\ell^{p}$ or $c_{0}$. To overcome this difficulty, we use the following Dvoretzky's theorem, which says that an infinite-dimensional Banach space contains an 'almostisometric' copy of $\ell_{m}^{2}$ for every $m \in \mathbb{N}$ (where $\ell_{m}^{2}$ denotes the space $\mathbb{R}^{m}$ equipped with the Euclidean norm).

THEOREM 8 (Dvoretzky's theorem). Let $X$ be an infinite-dimensional Banach space. Then, for every $\varepsilon>0$ and every $m \in \mathbb{N}$, there is an m-dimensional subspace $Y \subset X$ and an isomorphism $T: Y \rightarrow \ell_{m}^{2}$ such that $\|T\| \cdot\left\|T^{-1}\right\| \leq 1+\varepsilon$.

Proof. See the original paper [2] or the monograph [1].

The following proposition will be used to obtain our main result, but it is also interesting in its own right. It implies that, given one of the finite-dimensional subspaces whose existence is guaranteed by Dvoretzky's theorem (which says that $c=\|T\|$. $\left\|T^{-1}\right\| \leq 1+\varepsilon$ ), we can find a basis whose properties are very similar to the properties of the canonical basis of $\ell_{m}^{2}$ (where the statements $(i i)-(i i i)$ below are true with $c=1$ ).

THEOREM 9. Let $Y$ be an m-dimensional Banach space, $T: Y \rightarrow \ell_{m}^{2}$ an isomorphism and $c=\|T\| \cdot\left\|T^{-1}\right\|$. Then $Y$ has a basis $\left\{x_{1}, \ldots, x_{m}\right\}$ with the following properties:

(i) $\left\|x_{i}\right\|=1$ for every $i \in\{1, \ldots, m\}$,

(ii) $\left\|\sum_{i \in I} \alpha_{i} x_{i}\right\| \leq c\left\|\sum_{i=1}^{m} \alpha_{i} x_{i}\right\|$ for every $I \subset\{1, \ldots, m\}$ and $\alpha_{1}, \ldots, \alpha_{m} \in \mathbb{R}$,

(iii) $\left\|\sum_{i=1}^{m-1} \alpha_{i+1} x_{i}\right\| \leq c^{2}\left\|\sum_{i=1}^{m} \alpha_{i} x_{i}\right\|$ for every m-tuple $\alpha_{1}, \ldots, \alpha_{m} \in \mathbb{R}$.

Proof. Note that by replacing $T$ by a suitable multiple, we may assume that $\|T\|=1$ and $\left\|T^{-1}\right\|=c$. Let $e_{1}, \ldots, e_{m}$ be the canonical basis of $\ell_{m}^{2}$ and put

$$
x_{i}=\frac{T^{-1}\left(e_{i}\right)}{\left\|T^{-1}\left(e_{i}\right)\right\|}, \quad i \in\{1, \ldots, m\} .
$$

It is clear that $\left\|x_{i}\right\|=1$ for every $i \in\{1, \ldots, m\}$ and that $\left\{x_{1}, \ldots, x_{m}\right\}$ is a basis. Note that

$$
e_{i}=\left\|T^{-1}\left(e_{i}\right)\right\| T\left(x_{i}\right), \quad i \in\{1, \ldots, m\}
$$


Given an arbitrary $I \subset\{1, \ldots, m\}$ and $\alpha_{1}, \ldots, \alpha_{m} \in \mathbb{R}$, we have

$$
\begin{aligned}
& \left\|\sum_{i \in I} \alpha_{i} x_{i}\right\|=\left\|\sum_{i \in I} \frac{\alpha_{i} T^{-1}\left(e_{i}\right)}{\left\|T^{-1}\left(e_{i}\right)\right\|}\right\|=\left\|T^{-1}\left(\sum_{i \in I} \frac{\alpha_{i} e_{i}}{\left\|T^{-1}\left(e_{i}\right)\right\|}\right)\right\| \\
& \leq c\left\|\sum_{i \in I} \frac{\alpha_{i} e_{i}}{\left\|T^{-1}\left(e_{i}\right)\right\|}\right\|=c \sqrt{\sum_{i \in I} \frac{\alpha_{i}^{2}}{\left\|T^{-1}\left(e_{i}\right)\right\|^{2}}} \leq c \sqrt{\sum_{i=1}^{m} \frac{\alpha_{i}^{2}}{\left\|T^{-1}\left(e_{i}\right)\right\|^{2}}} \\
& =c\left\|\sum_{i=1}^{m} \frac{\alpha_{i} e_{i}}{\left\|T^{-1}\left(e_{i}\right)\right\|}\right\|=c\left\|\sum_{i=1}^{m} \alpha_{i} T\left(x_{i}\right)\right\|=c\left\|T\left(\sum_{i=1}^{m} \alpha_{i} x_{i}\right)\right\| \\
& \leq c\left\|\sum_{i=1}^{m} \alpha_{i} x_{i}\right\|
\end{aligned}
$$

To verify the third condition, note that for every $i \in\{1, \ldots, m\}$ we have

$$
1=\left\|e_{i}\right\|=\left\|T\left(T^{-1}\left(e_{i}\right)\right)\right\| \leq\|T\| \cdot\left\|T^{-1}\left(e_{i}\right)\right\|=\left\|T^{-1}\left(e_{i}\right)\right\|,
$$

i.e. $1 /\left\|T^{-1}\left(e_{i}\right)\right\| \leq 1$. Now, for any choice of $\alpha_{1}, \ldots, \alpha_{m} \in \mathbb{R}$, we obtain

$$
\begin{aligned}
& \left\|\sum_{i=1}^{m-1} \alpha_{i+1} x_{i}\right\|=\left\|\sum_{i=1}^{m-1} \frac{\alpha_{i+1} T^{-1}\left(e_{i}\right)}{\left\|T^{-1}\left(e_{i}\right)\right\|}\right\|=\left\|T^{-1}\left(\sum_{i=1}^{m-1} \frac{\alpha_{i+1} e_{i}}{\left\|T^{-1}\left(e_{i}\right)\right\|}\right)\right\| \\
& \leq c\left\|\sum_{i=1}^{m-1} \frac{\alpha_{i+1} e_{i}}{\left\|T^{-1}\left(e_{i}\right)\right\|}\right\|=c \sqrt{\sum_{i=1}^{m-1} \frac{\alpha_{i+1}^{2}}{\left\|T^{-1}\left(e_{i}\right)\right\|^{2}}} \leq c \sqrt{\sum_{i=1}^{m-1} \alpha_{i+1}^{2}} \\
& \leq c \sqrt{\sum_{i=1}^{m} \alpha_{i}^{2}} \leq c \max _{i \in\{1, \ldots, m\}}\left\|T^{-1}\left(e_{i}\right)\right\| \sqrt{\sum_{i=1}^{m} \frac{\alpha_{i}^{2}}{\left\|T^{-1}\left(e_{i}\right)\right\|^{2}}} \\
& \leq c^{2} \sqrt{\sum_{i=1}^{m} \frac{\alpha_{i}^{2}}{\left\|T^{-1}\left(e_{i}\right)\right\|^{2}}}=c^{2}\left\|\sum_{i=1}^{m} \frac{\alpha_{i} e_{i}}{\left\|T^{-1}\left(e_{i}\right)\right\|}\right\|=c^{2}\left\|\sum_{i=1}^{m} \alpha_{i} T\left(x_{i}\right)\right\| \\
& =c^{2}\left\|T\left(\sum_{i=1}^{m} \alpha_{i} x_{i}\right)\right\| \leq c^{2}\left\|\sum_{i=1}^{m} \alpha_{i} x_{i}\right\| \text {. }
\end{aligned}
$$

Choose an arbitrary $\varepsilon>0$. Given an infinite-dimensional space $X$, we can combine the previous theorem with Dvoretzky's theorem to see that the assumptions of Lemma 4 are satisfied (note that $\varepsilon$ might be arbitrarily large; we are using Dvoretzky's theorem only to ensure that the values $c=1+\varepsilon$ and $d=(1+\varepsilon)^{2}$ in Lemma 4 do not depend on $m$ ). Thus, we have proved the following corollary.

COROllary 10. Let $X$ be an arbitrary infinite-dimensional Banach space. Then $X$ does not have the property (JK).

Since we know that every finite-dimensional space has the property (JK), we arrive at the following conclusion. 
Corollary 11. A Banach space has the property (JK) if and only if it is finitedimensional.

Acknowledgement. The author thanks the anonymous referee whose suggestions helped to improve this paper.

\section{REFERENCES} 2006).

1. F. Albiac and N. J. Kalton, Topics in Banach space theory (Springer, New York, NY,

2. A. Dvoretzky, Some results on convex bodies and Banach spaces, in Proc. Int. Symp. Linear Spaces (Jerusalem, 1960) (Jerusalem Academic Press, Jerusalem, 1961) 123-160.

3. J. Jarník and J. Kurzweil, A general form of the product integral and linear ordinary differential equations, Czech. Math. J. 37 (1987), 642-659.

4. M. I. Kadets and M. G. Snobar, Some functionals over a compact Minkowski space, Math. Notes 10 (1971), 694-696 (translated to English from Mat. Zametki 10 (1971), 453-457).

5. Š. Schwabik, The Perron product integral and generalized linear differential equations, Časopis Pěst. Mat. 115 (1990), 368-404.

6. A. Slavík and Š. Schwabik, Henstock-Kurzweil and McShane product integration; descriptive definitions, Czech. Math. J. 58 (2008), 241-269. 